\title{
Effect of Precipitate Shape and Habit on Mechanical Asymmetry in Magnesium Alloys
}

\begin{abstract}
JOSEPH D. ROBSON, NICOLE STANFORD, and MATTHEW R. BARNETT
Asymmetric yield behavior in tension and compression is a common and usually undesirable feature of wrought magnesium alloys. To prevent yield asymmetry, it is necessary to favor slip over twinning, as it is the unidirectional nature of twinning combined with the strong textures produced in wrought magnesium alloys that produce yield asymmetry. In this article, the potential to use precipitates to strengthen selectively against twin growth is discussed. The effect of precipitate's shape and habit on strengthening of slip and twinning is calculated using simple Orowan-based models. It is shown that basal plate precipitates, although being poor strengtheners against basal slip, are good strengtheners against twin growth. This is because they produce the maximum unrelaxed back-stress when they remain unsheared inside the twin. The predictions of the model have been validated against experiments for two alloys that form different precipitate types: AZ91 (basal plates). and Z5 (c-axis rods). Crystal plasticity modeling has been used to predict that an optimized distribution of basal plate precipitates is expected to strongly reduce yield asymmetry, even in strongly textured magnesium alloy.
\end{abstract}

DOI: $10.1007 / \mathrm{s} 11661-012-1466-0$

(C) The Minerals, Metals \& Materials Society and ASM International 2012

\section{INTRODUCTION}

MeCHANICAL asymmetry is often observed in wrought magnesium products and is usually detrimental to performance. ${ }^{[1]}$ The origin of these effects is now well understood, which is due to the large differences in critical resolved shear stress (CRSS) for the deformation systems operating in the hexagonal crystal. As a result, the strength in directions in which easy deformation modes (basal slip and $\{10 \overline{1} 2\}$ c-axis tension twinning) can be activated is low compared with the strengths in the directions where the resolved shear stress for these systems is low. Another consequence of the relative ease of activation of basal slip and $\{10 \overline{1} 2\}$ twinning is that strong basal textures develop during thermomechanical processing of magnesium. ${ }^{[2]}$ This means that a loading direction that is in a weak orientation for one grain will be in a weak orientation for the entire grain assembly.

One solution to this issue, which has been the subject of recent intensive research, is to produce wrought products with randomized or non-basal texture, so that the grain population always contains a mixture of grains in weak and strong orientations regardless of the direction of the applied load. Various approaches have been used to modify the texture of wrought magnesium alloys; those that have been successful include adding rare-earth elements ${ }^{[3-5]}$ and using more complex strain

JOSEPH D. ROBSON, Reader, is with the Manchester Materials Science Centre, University of Manchester, Grosvenor Street, Manchester M1 7HS, UK. Contact e-mail: joseph.robson@manchester.ac.uk NICOLE STANFORD, Research Fellow, MATTHEW BARNETT, Professor, are with the Centre for Material and Fibre Innovation, Deakin University, Pigdons Road, Geelong 3217, Australia.

Manuscript submitted April 23, 2012.

Article published online October 17, 2012 paths. ${ }^{[6]}$ However, these methods have a number of disadvantages. Rare-earth additions are expensive, and using a more complex strain path during processing may not be practical.

This article explores the potential to use a different strategy to reduce yield asymmetry. Rather than changing the texture, the aim of the current study is to investigate whether the fundamental source of the problem - the large difference in CRSS of the different deformation modescan be addressed directly. This necessarily requires finding a method by which the easily activated deformation modes can be strengthened substantially more than the difficult to activate modes, so that the relative difference in CRSS is reduced. One potential route to provide this differential strengthening is to take advantage of the fact that precipitation in magnesium alloys is also highly anisotropic, and precipitate's shape and habit are expected to have a large effect on the strengthening of any given slip system. ${ }^{[7-9]}$

However, to reduce anisotropy and asymmetry, it is not sufficient to provide differential strengthening against slip alone because magnesium also deforms by twinning, and the $\{10 \overline{1} 2\}$ twinning mode in particular requires strong suppression to prevent its dominating yield behavior for loading conditions that produce c-axis tension. In a previous study, the authors have discussed the interaction between precipitates and $\{10 \overline{1} 2\}$ and suggested a simple semi-quantitative model for determining the effectiveness of a precipitate distribution in preventing twin growth. ${ }^{[8,9]}$ This model suggests that precipitate's shape and habit will also have a strong effect on the resistance that a precipitate distribution provides to twinning.

In this article, the role of precipitate's shape and habit on the resistance to slip (basal and prismatic) and $\{10 \overline{1} 2\}$ twinning are discussed. A simple model described previously ${ }^{[8]}$ and based on the Orowan equation is used to 
predict what precipitate's characteristics are likely to be effective in reducing asymmetry in strongly textured magnesium alloys. However, a calculation of the increment of Orowan strengthening alone is insufficient to estimate the effect of precipitates on asymmetry in a polycrystalline alloy because texture also needs to be considered. A key novel aspect of this study is that the Orowan calculation has been coupled with a proven plasticity model ${ }^{[10-12]}$ to provide a prediction of behavior for a realistic polycrystalline case as well as reveal the expected effects of different precipitate types on the relative deformation system activity. Finally, the model predictions are compared against experimental results for two alloys that form precipitates of distinctly different shapes and habits.

\section{ORIGIN AND ELIMINATION OF ASYMMETRY}

The typical extrusion texture for non rare-earth containing magnesium alloys is a strong $\langle 10 \overline{1} 0\rangle$ fiber texture in which the basal poles are distributed radially around the extrusion axis. This means that in axial tension or compression the resolved shear stress for basal slip, which is the easiest deformation mode in magnesium, is very low and other deformation modes control yield despite their higher CRSS. ${ }^{[13-15]}$ When tested in tension, it has been demonstrated that the activation of prismatic slip controls the yield stress. ${ }^{[13-15]}$ In compression, the grains are favorably oriented for activation of $\{10 \overline{1} 2\}$ twinning, and the CRSS for this mode is much less than prismatic slip in conventional magnesium alloys. ${ }^{[13-15]}$ Thus, in compression, $\{10 \overline{1} 2\}$ twinning activates and produces yield at a much lower stress than that needed to activate prismatic slip, hence producing mechanical asymmetry.

On the basis of the above discussion, it is clear that to reduce asymmetry, it is necessary to reduce the difference in the relative CRSS for prismatic slip and $\{10 \overline{1} 2\}$ twinning. This will be achieved if both modes are strengthened equally, which is one reason that strongly textured polycrystals of magnesium are less asymmetric than that would be expected from single crystal CRSS values. This is explained in detail elsewhere. ${ }^{[16]}$ However, to eliminate asymmetry, it is necessary to suppress $\{10 \overline{1} 2\}$ twinning more strongly than slip so that yield in compression becomes controlled by slip and not twinning. This means suppressing twinning, so that the CRSS for slip is reached before that for twinning when loading in axial compression.

For single crystal magnesium, the CRSS for prismatic slip has been reported to lie in the range 39 to $50 \mathrm{MPa}$, and that for $\{10 \overline{1} 2\}$ twinning is $\simeq 2 \mathrm{MPa} .^{[16,17]}$ The CRSS ratio (prismatic/twinning) is thus $\simeq 20$. To activate prismatic slip before twinning for a single crystal loaded in a $\langle 10 \overline{1} 0\rangle$ direction, this ratio needs to be less than 0.87 , which could only be achieved by increasing the CRSS for twinning by over 20 times without strengthening against prismatic slip. It is difficult to envisage a mechanism in single crystals, which could produce the necessary level of differential strengthening.
In polycrystals, which are of much more practical interest, the situation is further complicated, but the requirement for differential strengthening is reduced. First, the strengthening of all modes obtained in polycrystals due to grain boundaries and grain/grain interactions leads to a reduction in the relative differences in CRSS values. ${ }^{[16]}$ For example, for AZ31 polycrystals, plasticity models suggest that the CRSS for prismatic slip is $77 \mathrm{MPa}$ and for $\{10 \overline{1} 2\}$ twinning is $33 \mathrm{MPa},{ }^{[18]}$ giving a CRSS ratio of 2.3 (almost 10 times less than in the single crystal case). The strengthening effect on prismatic slip due to grain size effects is discussed in more detail in Reference 19. Second, another significant factor is the texture spread in polycrystals. Even in strongly textured extrusions, there is a significant spread in orientations around the ideal texture fiber. This spread will influence the relative increase in CRSS needed to suppress twinning during compression because basal slip on grains that have a non-zero Schmid factor for this mode can also play a role in accommodating deformation.

In determining how twinning might be strengthened more potently than prismatic slip, it is necessary to recognize that twinning and slip are not independent processes, but that the nucleation and growth of a twin require slip dislocation activity. The nucleation stage of $\{10 \overline{1} 2\}$ twin formation involves local reactions of slip dislocations to form the necessary twinning dislocations at sites of stress concentration such as grain boundaries. ${ }^{[2-23]}$ The growth of a twin produces internal stresses that exceed the yield strength of the matrix and are thus accommodated by plastic deformation processes including slip, kinking or double twinning. ${ }^{[24]}$ These processes will themselves be influenced by the presence of second phase particles.

To calculate the total strengthening effect against twin growth when precipitates are present is a complex problem because, in addition to any precipitate effect, there is also hardening due to other mechanisms such as the Hall-Petch effect and parent dislocation transmutation in the twin. ${ }^{[25]}$ Although models are now available for these other hardening effects, ${ }^{[25,26]}$ it is not obvious how these can be adapted to include the effect of precipitates. In this study, a simpler, semi-quantitative approach is taken by modifying the effective CRSS for $\{10 \overline{1} 2\}$ twinning derived from model calibration in a precipitate-free case to consider the additional increment in CRSS that would be expected from precipitates. It is important to note that this study considers only behavior at low ( $<1.5 \mathrm{pct})$ strains (i.e., just after yield) before significant work hardening has occurred. Therefore, the complex effects of precipitates on the accommodation mechanisms that will necessarily operate as the accumulated strain, and hence twin fraction increases are neglected.

\section{A. Crystal Plasticity Modeling}

The texture effect in polycrystals may be explored using crystal plasticity modeling. In this study, the well-proven self-consistent viscoplastic (VPSC) model developed by Tomé and Lebensohn (e.g., References 10-12) was used. 


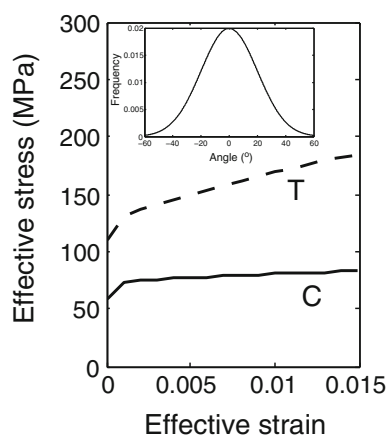

(a)

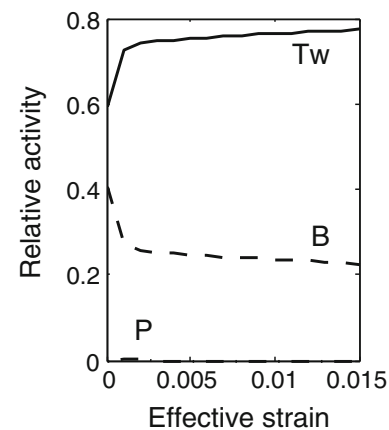

(d)

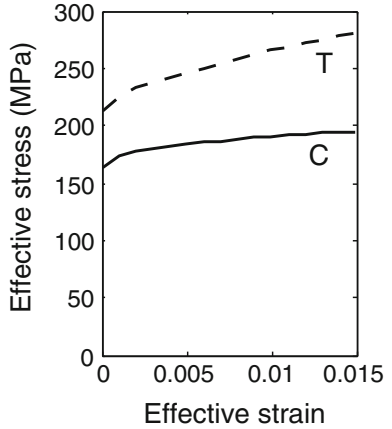

(b)

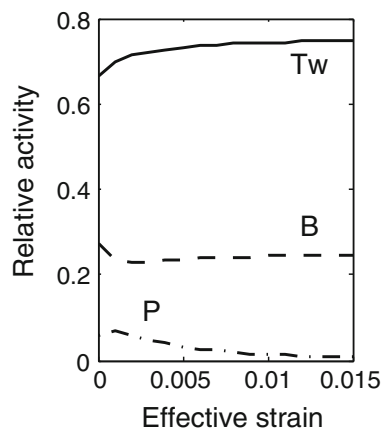

(e)

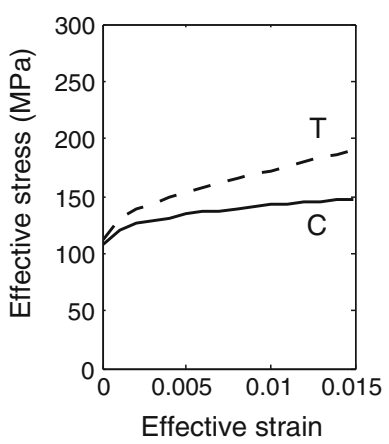

(c)

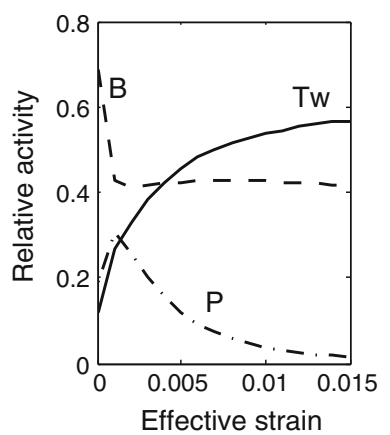

(f)

Fig. 1-Predicted stress-strain response for deformation in compression (C) and tension (T) (a) standard (AZ31) CRSS values [inset: (0001) pole figure showing input texture, loading direction indicated]. (b) All CRSS values increased by $50 \mathrm{MPa}$. (c) CRSS for \{1012\} twinning only increased by $50 \mathrm{MPa}$. (d) through (e) Predicted relative activity of deformation systems for cases [(a) through (c)] respectively.

This model has been widely and successfully applied to calculate mechanical behavior and texture evolution in magnesium alloys and has correctly predicted asymmetric flow behavior in magnesium alloy extrusions. ${ }^{[18,27]}$ Full details of this model as applied to magnesium alloys are given elsewhere. ${ }^{[18,28]}$

Inputs to the simulation are the imposed deformation, initial texture, and CRSS/hardening behavior of the individual deformation systems. By varying the CRSS values for the critical deformation modes that control asymmetry, the model can be used to predict the relative strengthening required for eliminating yield asymmetry. The model was first run for a standard case using a set of CRSS values proven for AZ31 ${ }^{[18]}$ and 500 grains using a simulated basal extrusion texture with 40 degrees (full width half maximum) orientation spread normally distributed around the perfect fiber. The probability distribution function showing the texture spread is presented in Figure 1(a, inset) and simulates what would be typically be reported as a strong basal texture when observed in a commercial magnesium alloy. ${ }^{[29]}$ All simulations were run with a loading direction that corresponds to the axial direction of an extrusion with a strong basal fiber texture - i.e., the loading direction is perpendicular to the directions with the highest intensity of basal plane normals.

The predicted flow stress evolution under axial tension and compression for the standard (AZ31) case is shown in Figure 1(a). As expected, the VPSC model predicts that the flow stress in compression is much lower than that in tension. The predicted asymmetry ratio (compressive/tensile flow stress) at zero equivalent plastic strain is 0.53 , which is comparable to that measured in strongly textured AZ31 extrusions. ${ }^{[29]}$

Figure 1(b) shows the effect of strengthening all deformation systems equally. In this simulation, the CRSS of all systems were increased by $50 \mathrm{MPa}$. This increase is a reasonable estimate of the maximum strengthening provided by precipitation according to Orowan calculations. $^{[7,8]}$ The predicted effect of strengthening all systems is to shift both the tensile and compressive flow stresses to higher values. As a result, the asymmetry ratio is reduced to 0.77 (at an effective plastic strain of zero). This demonstrates the point made by Hutchinson and Barnett ${ }^{[16]}$; any mechanism that strengthens all systems equally will necessarily reduce the asymmetry ratio.

For the final simulation, only the CRSS for $\{10 \overline{1} 2\}$ twinning was increased (by $50 \mathrm{MPa}$ ). This means that for this simulation, the CRSS for twinning was $83 \mathrm{MPa}$, which is slightly greater than that for prismatic slip (which is kept at $77 \mathrm{MPa}$ ), giving a relative CRSS ratio of 1.08 . The predicted flow curves in this case are shown in Figure 1(c). The effect of suppressing twinning is to strongly increase the compressive flow stress, whereas the tensile flow stress is unaffected (compared to the standard case in (a)). The net result is an almost complete elimination of asymmetry (the predicted asymmetry ratio is 0.96 at zero equivalent plastic strain). This simulation demonstrates that even in the case of a strong basal texture, yield asymmetry can be reduced or eliminated by differential strengthening to promote slip over twinning.

Another output of the VPSC model is the relative activity of each system in accommodating the imposed 
deformation, Figures 1(d) through (f) shows the relative slip system activity corresponding to the compressive deformation for the cases shown in Figures 1(a) through (c) respectively. It can be seen that in both the standard (a, d) and equal strengthening case (b, e), $\{10 \overline{1} 2\}$ twinning is predicted to be responsible for accommodating most of the strain in the early stages of deformation, with basal slip playing a secondary role and only a small contribution from prismatic slip. When twinning is reduced by selectively strengthening this system (c,f), it no longer dominates the early stages of deformation. The simulation results suggest that both basal and prismatic slip instead make a greater contribution the imposed deformation. Basal slip can make an important contribution because there are sufficient grains tilted enough to give activation of this mode. The simulation also suggests that as strain increases, twinning will have to play an increasing role. Prismatic slip makes a reducing contribution to deformation as the fraction of twinned material increases because material in the twin is not in a favorable orientation for prismatic slip. The simulation suggests that even when a more symmetric yield response is obtained, asymmetry may reappear as texture evolves at higher strain. This would be undesirable during a forming operation. However, in cases where the reduction of yield asymmetry is of primary importance, the model suggests selective strengthening to suppress twinning can be highly effective.

A key question is therefore how can this differential strengthening be obtained? Solute additions are one possibility, as it is known that solute does not harden all systems equally and indeed may even soften some systems (e.g., the softening effect of zinc on prismatic slip reported for single crystals of magnesium ${ }^{[30,31]}$ ). However, it is unlikely that solute can provide the large differential strengthening needed to eliminate asymmetry. Strengthening precipitates, however, may be able to produce this effect. In particular, as already demonstrated by Nie, ${ }^{[7]}$ precipitate's shape and habit has a profound effect on the strengthening against basal slip. A similar effect is also expected for other deformation modes. ${ }^{[8,9]}$ By designing an alloy system with precipitates that provide the desired differential strengthening effect, a significant reduction or even elimination of yield asymmetry should be achievable.

\section{EFFECT OF PRECIPITATES ON STRENGTHENING}

\section{A. Hardening Against Slip}

In a previous publication, the effect of precipitate's shape and habit on strengthening against basal and prismatic slip was considered. ${ }^{[8,9]}$ This analysis was based on the application of the Orowan equation to calculate stress required to bow dislocations around precipitates and a statistical approach to determine the mean number density and area of precipitates on a given type of slip plane. The method is an extension of that first applied to magnesium alloys by Nie, ${ }^{[7]}$ where only basal slip was considered. This analysis in turn is based on earlier study on age hardenable aluminium alloys which also form precipitates in the form of plates or rods on rational habit planes (e.g., References 32-34).

The general form of the Orowan equation used in this study can be written in the form: ${ }^{[35]}$

$$
\Delta \tau=\left(\frac{G b}{2 \pi \sqrt{1-v}}\right)\left(\frac{1}{\lambda}\right) \log \left(\frac{D_{\mathrm{p}}}{r_{0}}\right)
$$

where $\Delta \tau$ is the increase in CRSS due to precipitate strengthening, $G$ is the shear modulus, $b$ the Burgers vector of the gliding dislocations, $v$ the Poisson ratio, $\lambda$ the effective inter-particle spacing on the slip plane, $D_{\mathrm{p}}$ the mean planar diameter of the particles on the slip plane, and $r_{0}$ the dislocation core radius.

In Reference 8, three particle types were considered: basal plates, c-axis rods, and randomly distributed spheres (which were used as an isotropic reference). In the current article, the analysis is extended to prismatic plates. These habits and morphologies cover all of the precipitate types most commonly observed in commercial magnesium alloys. ${ }^{[7]}$ The analysis for a given precipitate type requires finding the appropriate values of $\lambda$ and $D_{\mathrm{p}}$ to use in Eq. [1]. The general method used is outlined below, with specific details for each precipitate type provided elsewhere for basal ${ }^{[7]}$ and prismatic slip. ${ }^{[8]}$

The spacing between precipitates is found by first determining the number of precipitates intercepted per unit area on a given slip plane. To do this, the method of Fullman $^{[36]}$ is followed. First, the probability of cutting a single randomly positioned precipitate in a unit volume is calculated. This depends on the length of the precipitate resolved in the direction normal to the slip plane. For example, for a plate-shaped precipitate oriented perpendicular to the slip plane, the resolved length in the plane normal direction corresponds to the plate diameter, and the probability of interception is high. However, for plates oriented parallel to the slip plane (e.g., plates lying on the basal plane for basal slip), the resolved length is the precipitate thickness, and the probability of interception is therefore low. Once the probability of intercepting a single precipitate is known, the number of precipitates intercepted per unit area of slip plane $\left(N_{\mathrm{A}}\right)$ can be calculated from geometry. The mean spacing (center-to-center) distance is then simply given by $1 / \sqrt{N_{\mathrm{A}}}$.

The mean planar diameter of the particles on the slip plane is also found following the analysis of Fullman. ${ }^{[7,36]}$ Rods and plates will produce either circular, elliptical, or rectangular cross sections when intersected by a slip plane depending on the plane orientation. The mean planar diameter is found by determining the average length of the precipitate parallel to the direction of the gap to be bowed by the dislocations.

Figure 2 shows the calculated Orowan stress for (a) basal and (b) prismatic slips for basal plates, c-axis rods, prismatic plates, and spherical particles (used as a reference). This calculation was performed assuming a precipitate's volume fraction of 5 pct, with the aspect ratio of plates being defined as $t_{\mathrm{t}} / d_{\mathrm{t}}=0.1$, where $t_{\mathrm{t}}$ is 


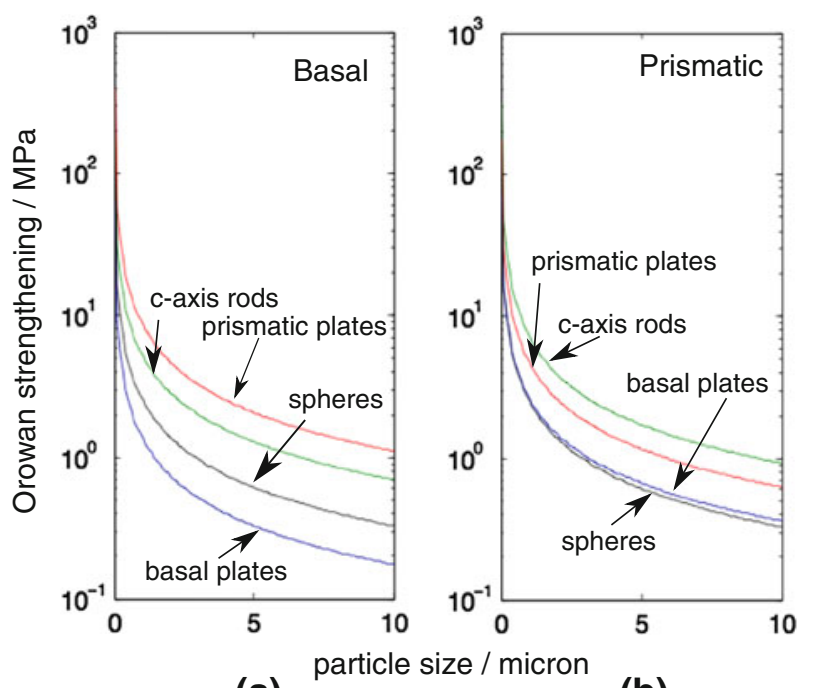

(a)

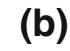

Fig. 2-A calculation of the Orowan stress required to bypass basal plates, prismatic plates, c-axis rods, and spherical precipitates for $(a)$ basal slip $(b)$ prismatic slip. Particle volume fraction $=5$ pct, aspect ratio of plates $=0.1$, and aspect ratio of rods $=10$.

the plate thickness, $d_{\mathrm{t}}$ is the plate diameter, and the aspect ratio of rods $l_{\mathrm{t}} / d_{\mathrm{t}}=10$ with $l_{\mathrm{t}}$ as the rod length. These represent typical values for an age hardening magnesium alloy and are used throughout the article to highlight the important trends. Application to some specific alloys is discussed later. In this, and subsequent plots, the $x$-axis gives the effective particle's dimension, which is defined as $V_{\mathrm{p}}^{1 / 3}$ where $V_{\mathrm{p}}$ is the particle volume. This means that a given $x$-value corresponds to the same particle volume for plates, rods, and spheres. It is plotted this way to facilitate comparison between different shapes and habits at the same number and total volume fraction of particles.

The calculations confirm that basal plates are poor strengtheners against basal slip. This is because the probability of such a plate being intersected by a given basal plane is very low, leading to a large inter-particle spacing on the plane. Prismatic plates are predicted to be the best strengtheners against basal slip because they are in the ideal orientation to intersect the basal slip plane. Furthermore, because there are three distinct prismatic plate orientations, a majority of the plates are always oriented to present a relatively large average effective planar diameter on the slip plane. This means that the gap through which a dislocation must bow is small, leading to high Orowan stress. For prismatic slip, basal plates are again predicted to be poor strengtheners. Although they are favorably oriented to intersect prismatic planes, because there is only one unique basal plate orientation, there is a large gap between precipitates in the c-axis direction (precipitate thickness direction) through which dislocations can bow. Prismatic plates provide better strengthening against prismatic slip, but c-axis rods are predicted to provide the most potent strengthening against this mode for the choice of volume fraction, size, and aspect ratio used here. The effect of varying these parameters is discussed elsewhere. ${ }^{[9]}$

\section{B. Hardening Against Twinning}

It is now clear from several experimental studies that particles can have a strong effect on twinning in magnesium. ${ }^{[37-43]}$ However, the details of the interaction between precipitates and twins have received only limited attention in the literature and are still not well understood. ${ }^{[23]}$

At least three different behaviors have been observed when twins interact with particles in magnesium alloys. Large particles can often completely arrest twin growth, often with twinning continuing by nucleation of a new twin in the matrix on the far side of the particle. ${ }^{[39]}$ Alternatively, particles can become engulfed by a growing twin without themselves suffering shearing. ${ }^{[39,40,43]}$ Finally, it has also been observed that particles can be sheared inside the twin. ${ }^{[37,42]}$ The type of behavior observed appears to depend on precipitate's size and shape. Shearing of precipitates by twins has only been observed in alloys containing very fine or coherent precipitates. ${ }^{[37,42]}$ For precipitates of the sizes formed after typical aging treatment, particles often seem to become engulfed by a twin without shearing themselves. ${ }^{[39,43]}$ The particles will, however, attempt to accommodate part of the twinning shear by undergoing a rigid body rotation once inside the twin. ${ }^{\text {[39] }}$

Recent study on AZ91 containing basal plate precipitates has revealed several interesting features commonly observed when a $\{10 \overline{1} 2\}$ twin interacts with particles. ${ }^{[43]}$ Selected TEM micrographs from this study are repeated in Figure 3. Full details of the experimental method and results are reported elsewhere. ${ }^{[43]}$ Figure 3(a) shows an example of a plate shaped precipitate that has been partly engulfed in a twin (marked T). The diffraction pattern in (b) contains reflections from both the matrix and twin demonstrates that the twin is of the expected $\{10 \overline{1} 2\}$ type, resulting in $86 \mathrm{deg}$ reorientation of the lattice. It can be seen that the precipitate is deflected when it enters the twin, and the angle of deflection is around $2.5 \mathrm{deg}$. This deflection is significantly less than $3.8 \mathrm{deg}$, which is the expected angle if the precipitate had sheared along with the matrix in the twin. The diffraction pattern in (c) also confirms the precipitate has not undergone the full twinning shear. These observations are consistent with previous study investigating the same precipitate type that also demonstrated that the precipitates are not sheared, but do undergo a rigid body rotation inside the twin. ${ }^{[39]}$

Figure 3(d) gives an example of another common observation, showing a twin boundary that has become deflected when it intersects particles. This is an indication that the particles are providing a resistance to migration of the boundary. Finally, Figure 3(e) shows a case where the tip of a growing twin has been arrested by a particle.

\section{Orowan and Back Stress}

Twinning involves nucleation and growth, and precipitates will influence both processes. Experimentally, it has been demonstrated in a number of precipitate containing magnesium alloy systems that the particles 

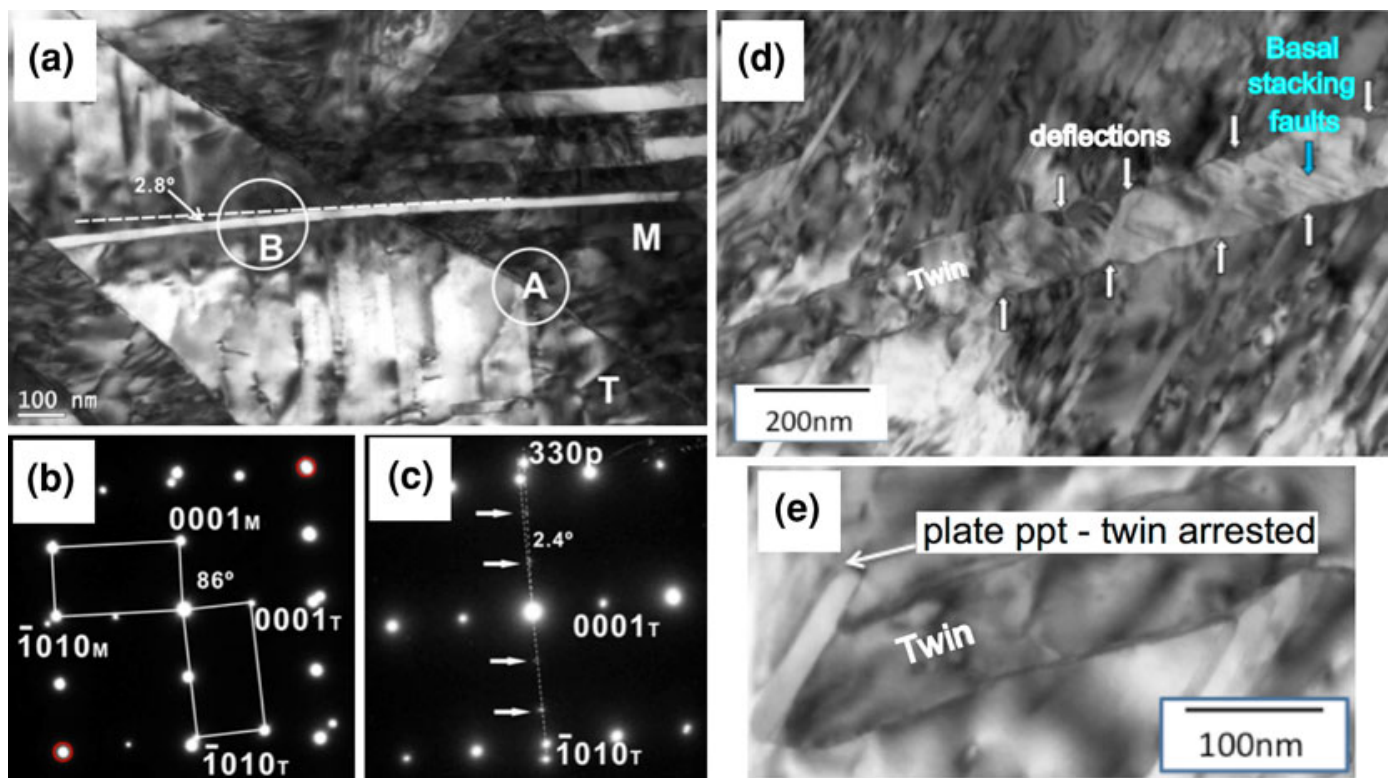

Fig. 3-TEM micrographs showing the interaction of precipitates with $\{10 \overline{1} 2\}$ twins in peak aged AZ91. (a) Deflection of a single precipitate plate as it enters a twin. (b) Diffraction pattern showing reorientation of lattice in twin. (c) Diffraction pattern of precipitate and matrix in twin demonstrating precipitate does not undergo full twinning shear. (d) Deflections in twin boundary where it intersects precipitates. (e) Twin tip arrested at precipitate.

do not prevent twin nucleation, but do lead to narrower twins (for a given applied strain). ${ }^{[40,41,43]}$ Indeed, in some cases, having particles seems to increase the number of twins (whilst decreasing their thickness). ${ }^{[40]}$ This is consistent with the supposition that by increasing the stress required to thicken a twin (in the grain interior) grains favorably oriented for twinning carry a greater load, leading to a higher stress concentrations in grain boundary regions where twins usually nucleate. ${ }^{[44]}$

An important observation is that precipitates are never observed to completely suppress twin nucleation, even in alloys with a high particle fraction. ${ }^{[41,43]}$ This may be because the local dislocation events that can occur to produce twin nuclei in grain boundary regions are not strongly influenced by precipitates because these regions are often precipitate free, although this requires further investigation. In this study, it is the additional stress for twin growth that is considered the critical factor in determining the strengthening against twinning, but it is still possible that changes in the critical stress for nucleation may be important.

To quantify the effect that precipitates may have in strengthening against twin growth, it is necessary to consider the addition stress that will be required to propagate a twin through a precipitate containing matrix. In a previous study, the contributions to this stress for an unsheared precipitate have been estimated. ${ }^{[8]}$ The two most important contributions arise from the Orowan stress required to bow twinning dislocations around the precipitate and the long-range back-stress due to the strain incompatibility between the sheared material in the twin and the unsheared particle.

Previously, ${ }^{[8]}$ it has been demonstrated that the Orowan stress required to bow the twinning dislocation is predicted to be very small. This is because the Orowan stress is proportional to the Burgers vector of the dislocation, and the twinning partial dislocations are characterized by a very short Burgers vector. ${ }^{[23]}$ For a typical particle size and spacing, the Orowan stress to bow the twinning dislocation is estimated as only 5 to $10 \mathrm{MPa}^{[8,43]}$ and this is too small to explain the observed increase in strength noted on precipitate formation in age hardenable magnesium alloys tested in a direction where yield is controlled by twinning. ${ }^{[43]}$

The other contribution to strengthening against twin growth comes from the additional back-stress on the twin due to the unsheared particles being embedded in sheared (twinned) material. This is an additional contribution to the overall back-stress that acts on the twin. ${ }^{[23]}$ Back-stress calculations based on the assumption that accommodation of the strain incompatibility is entirely elastic have been presented previously for the precipitate morphologies under consideration in this article $^{[8]}$ A plot of the calculated back-stress as a function of particle fraction is shown in Figure 4. There are two key points to be drawn from this plot; the first is that the back-stress is highly dependent on precipitate's shape and habit, with basal plates giving the highest value. The second is that the predicted magnitude of the back-stress under the assumption of completely elastic accommodation is very large for reasonable precipitate fractions (e.g., 5 to $10 \mathrm{pct}$ ) and exceeds the yield stress of the matrix. This implies that the strain incompatibility will not be accommodated purely elastically, but will also be relaxed by local plastic deformation around the particles.

It is thus considered that the dominant resistance nonsheared particles provide to twin growth arises from the additional back-stress in the twin, and the level of backstress sustained will depend on both the particle 


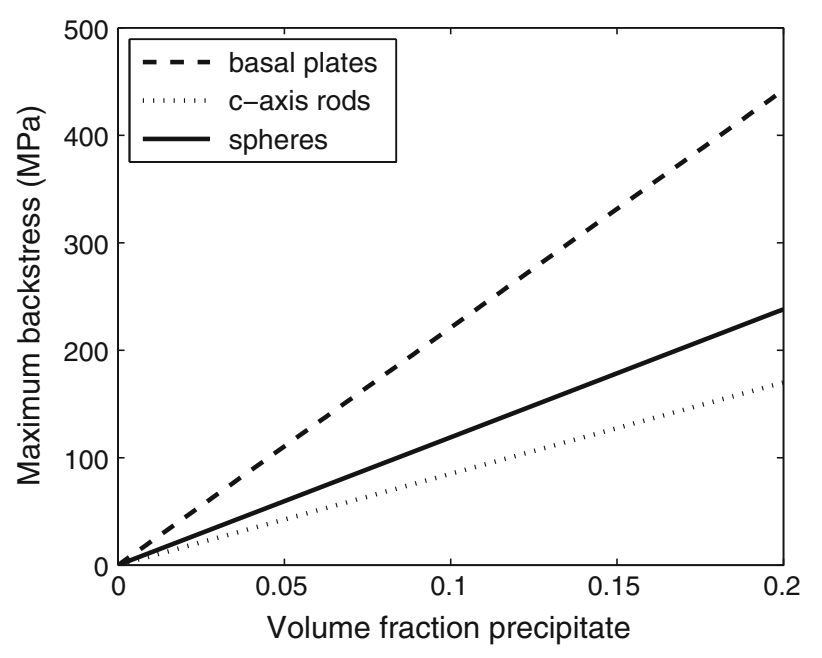

Fig. 4-A calculation of the back-stress produced by accommodation of non-deforming particles of different shape in a $\{10 \overline{1} 2\}$ twin, assuming accommodation occurs entirely elastically.

morphology and level of plastic relaxation that can occur. A further complication arises because the plastic relaxation in the twin will itself be influenced by the presence of precipitates in the twin that inhibit the necessary dislocation motion. Therefore, the resistance particles provide to plastic deformation in the twin is an important parameter that is expected to correlate directly with the strengthening against twin growth.

\section{Basal Slip in the Twin}

It is reasonable to assume that basal slip is responsible for the majority of the plastic deformation necessary to moderate the strain incompatibility between non-shearing particles and the sheared matrix in the twin. This is because the CRSS for basal slip is far less than that for other slip modes. As demonstrated previously, the Orowan calculation for basal slip can be repeated considering precipitates now embedded in twinned material. It is assumed that the particles are not sheared by the twin. Note that the reorientation of the lattice in the twin by $86 \mathrm{deg}$ means that basal plates in the parent have a prismatic like orientation in the twin (but lie only on one set of parallel prism planes), parent $\mathrm{c}$-axis rods lie in the basal plane in the twin, and prismatic plates in the parent lie on basal and pyramidal planes in the twin. These orientations are shown in sketch form in Figures 5(a) through (c). Figure 5(d) shows the calculated Orowan strengthening against basal slip in the twin.

As Figure 5(d) shows, the reorientation of the matrix in the twin has a profound effect on the strengthening contribution expected from the different precipitate populations. Basal plates, poor strengtheners in the parent material, are predicted to be the best strengtheners in the twin as they all oriented perpendicular to the basal plane in the twin, and being therefore in the best orientation to block basal slip. Prismatic plates are predicted to remain the potent strengtheners in the twin,

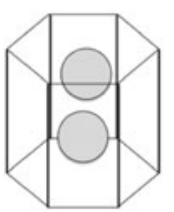

(a)

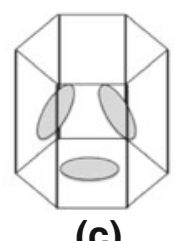

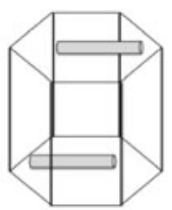

(b)

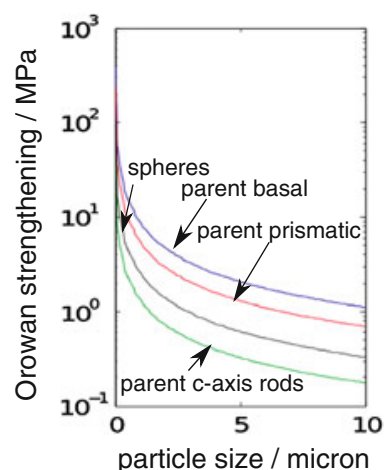

(d)
Fig. 5-Schematic showing orientation of precipitates in twinned material with respect to the hexagonal magnesium unit cell $(a)$ basal plates $(b)$ c-axis rods (c) prismatic plates. (d) A calculation of the Orowan stress inhibiting basal slip for basal plates, prismatic plates, c-axis rods, and spherical shaped precipitates in twinned material. Particle volume fraction $=5$ pct, aspect ratio of plates $=0.1$, and aspect ratio of rods $=10$.

and again this can be attributed to the three distinct orientations, so that (on average) $2 / 3$ of the precipitates are always in a good orientation to block basal slip in the twin. Parent c-axis rods are predicted to be very poor strengtheners against basal slip in the twin, as they lie in the basal plane and are therefore in a poor orientation to intersect basal slip planes.

\section{E. Effect on Asymmetry}

It has been demonstrated theoretically that particle shape and habit are expected to lead to differential strengthening against slip and twinning. It has also been shown that differential strengthening is a potential route to reduce or eliminate asymmetry. As already discussed, to achieve this goal, it is necessary to strengthen more strongly against twinning than prismatic slip.

Inspection of the calculations from the previous section suggests that of the precipitate types considered, basal plates best meet the required criterion. They strengthen relatively weakly against basal and prismatic slip in the parent, but provide the maximum back-stress and inhibition of plastic relaxation in the twin. To verify this, a simple model that provides a semi-quantitative estimate of the effect of different types of precipitate on asymmetry has been developed.

For this analysis, it is assumed that the inhibition of twin growth scales in direct proportion to the Orowan stress for plastic relaxation of the back-stress due to precipitates in the twin; i.e., if plastic relaxation is difficult, a high backstress will be sustained, and twin growth will be more difficult. As the CRSS for basal slip is much lower than that for other systems, even when precipitates are present, in practice, it is always predicted that plastic relaxation will occur when the back-stress reaches a value sufficient to activate basal slip in the twin.

It is further assumed that other possible slip and twinning modes (e.g., $\langle c+a\rangle$ slip) have negligible influence on yield, which is reasonable at room temperature in the magnesium alloys considered here. ${ }^{[1]}$ 
Although this is clearly an over-simplification it does provide a useful qualitative tool for determining the likely effect of a particular particle morphology and habit on yield asymmetry. As has already been demonstrated, for the case of a perfect basal texture, it is the ratio of the CRSS for prismatic slip to that for twinning that is critical in determining asymmetry. If a strengthening mechanism decreases this ratio, then asymmetry will be reduced. If this ratio is increased, asymmetry will be increased. As already shown, the value of this CRSS ratio for AZ31 (taken as representative of a magnesium alloy without precipitates) is 2.3 .

Figure 6 shows the predicted variation of this ratio as a function of precipitate volume fraction (at a constant equivalent precipitate size of $1 \mu \mathrm{m}$ ). As expected, basal plates are predicted to give the strongest reduction in

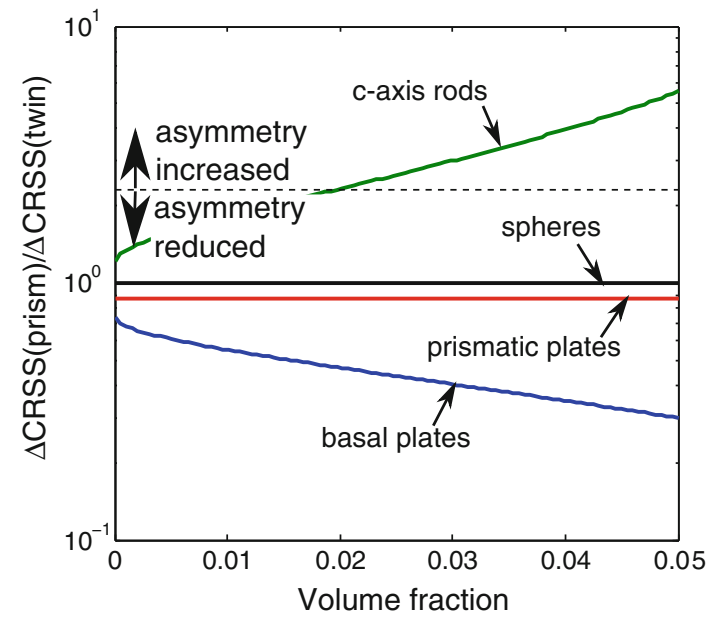

Fig. 6-A calculation of the ratio of the increase in critical resolved shear stress (CRSS) for prismatic slip compared to $\{10 \overline{1} 2\}$ twin growth for basal plates, prismatic plates, c-axis rods, and spherical particles. A value of this ratio $>2.3$ is predicted to lead to an increase in asymmetry on precipitation. Particle volume fraction $=5$ pct, aspect ratio of plates $=0.1$, aspect ratio of rods $=10$. asymmetry. Prismatic plates and spheres also are always predicted to reduce asymmetry. In the case of c-axis rods, it is predicted there is a transition at a critical volume fraction (which will depend on the precipitate size) above which precipitation is predicted to increase asymmetry. This is because above a critical volume fraction, c-axis rods are predicted to strengthen against prismatic slip more strongly than against twinning.

\section{EXPERIMENTAL VALIDATION}

The models developed in this study have made some specific predictions regarding the effect of different precipitate's shape and habit on asymmetry in strongly textured magnesium alloys. In particular, it has been predicted that basal plate shaped precipitates should produce a strong reduction in asymmetry, whereas c-axis rod precipitates may increase asymmetry. To test these predictions, experiments were performed on two alloys that form different precipitate types; AZ91 and Z5. These alloys were extruded and both produced strong basal textures. Note that the aging treatment does not change the texture or grain structure for either AZ91 or Z5 as the temperatures in both cases are too low. The alloys were then subject to axial tension and compression tests in the as-extruded condition (precipitate free) and peak aged condition (without prior solution treatment to avoid any changes to the grain structure or texture). Full details of the experiments are given elsewhere. ${ }^{[40,43]}$

Stress-strain curves for both alloys are shown in Figure 7. From these, the tension/compression asymmetry values (compressive 0.2 pct offset stress/tensile 0.2 pct offset stress) have been calculated and are shown on the figure. In the as-extruded case (prior to precipitation), the asymmetry values for both alloys are quite similar, and the compressive strength is between half and three quarters that of the tensile strength. On aging, the asymmetry of the AZ91 extrusion is reduced greatly,

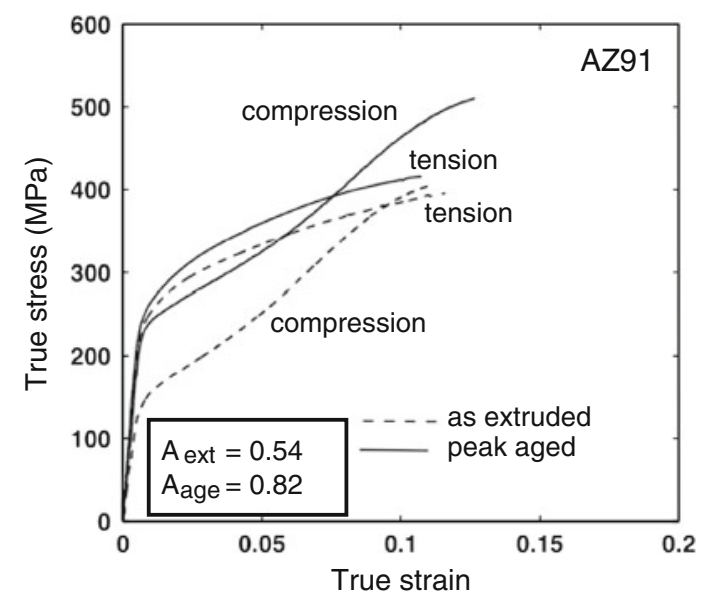

(a)

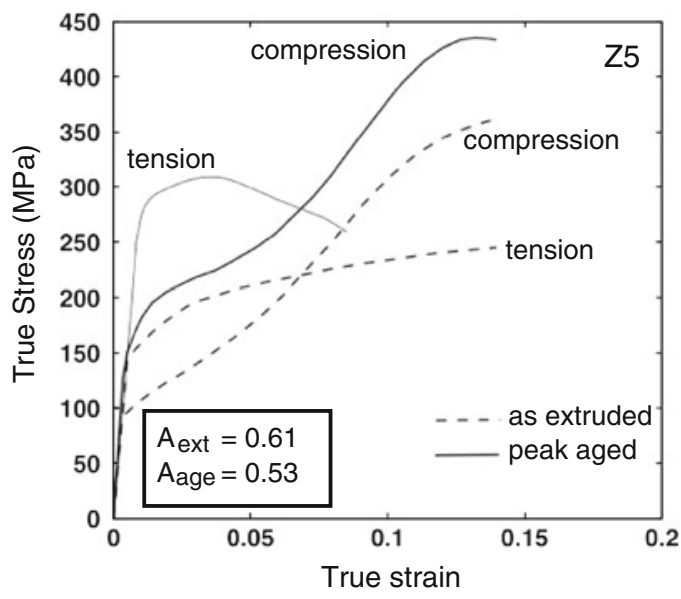

(b)

Fig. 7-Mechanical properties of the AZ91 and Z5 alloy extrusions. (a) Stress-strain curves for AZ91 tested in axial compression and tension in the aged and as-extruded condition. Asymmetry ratios $(A)$ for as-extruded (ext) and peak aged (age) indicated on plot. (b) Curves for Z5 in the aged and as-extruded condition. 
which is mainly due to an increase in the compressive yield strength. In Z5, however, the opposite is observed, and aging leads to an increase in asymmetry. The reason for this is that the tensile yield stress is strongly increased on precipitation but the compressive yield strength increases much less.

These observations are in full agreement with the predictions and are consistent with the inference that the basal plates in AZ91 are highly effective at inhibiting twin growth and hence raise the compressive yield stress, whereas the c-axis rods in Z5 strengthen against prismatic slip more effectively than against twin growth and hence strongly increase the tensile yield stress.

\section{DISCUSSION}

This study has demonstrated that in addition to manipulating texture, yield asymmetry in magnesium alloys may also be reduced by using a strengthening mechanism that can provide tailored resistance to inherently asymmetric deformation modes (i.e., twinning). It has also been shown that precipitates of different shape and habit provide such a differential strengthening effect and for the purposes of strengthening against growth of $\{10 \overline{1} 2\}$ twins, basal plate shaped precipitates are predicted to be most effective. These predictions have been shown to be qualitatively in agreement with experimental observations.

It is now possible to combine the predictions of the Orowan strengthening model with the VPSC model discussed earlier to predict whether under ideal conditions precipitation may be able to completely suppress asymmetry in a magnesium alloy extrusion with a strong basal texture. To provide the maximum strengthening effect and minimum asymmetry, it is necessary to maximize the volume fraction of basal plate precipitates and minimize the inter-particle spacing. Adding a dopant to $\mathrm{Mg}-\mathrm{Al}$ alloys, such as calcium, has been shown to be effective in refining the $\beta-\mathrm{Mg}_{17} \mathrm{Al}_{12}$ basal plates $^{[45]}$ as well as suppressing discontinuous precipitation (which is a further complication not considered here). Therefore, for the basis of this "best case" calculation, it is assumed that a volume fraction of 10 pet of randomly distributed basal plate precipitates of $50 \mathrm{~nm}$ equivalent size and aspect ratio 10 might be realistically achieved by careful alloy design and processing. Using this parameter set, the Orowan model was used to calculate the strengthening predicted for the deformation modes considered in this article, and these modified CRSS values were input into the VPSC model.

For these input parameters, the strengthening model predicts CRSS values for basal slip, prismatic slip, and $\{10 \overline{1} 2\}$ twinning of 67,140 , and $143 \mathrm{MPa}$, respectively. The value of $143 \mathrm{MPa}$ for twinning is resulted from adding the (unrelaxed) back-stress due to precipitates (110 $\mathrm{MPa}$ ) to the CRSS for twinning in the precipitatefree case $(33 \mathrm{MPa}){ }^{[18]}$ Pyramidal slip is also considered in the VPSC model. However, this mode was not predicted to be active in any of the simulations here, even if a lower bound is used for the strengthening of this system (which gives a CRSS of $208 \mathrm{MPa}$ ). Note also that the hardening parameters in the VPSC model have not been modified to include the effect of precipitates, and so the work hardening response at higher strain levels has not been captured.

Using these parameters, the predicted response in axial tension and compression, and the relative activity of each deformation mode is shown in Figure 8. It can be seen that asymmetry is strongly reduced in these predictions with an asymmetry ratio of 0.93 at zero equivalent plastic strain. Figure $8(\mathrm{~b})$ shows the predicted relative activity of the operating deformation modes in compression, and Figure 8(c) in tension. In compression, twinning is not predicted to be completely suppressed, and twinning, prismatic slip, and basal slip all make significant contributions to accommodating deformation. In tension, prismatic slip is predicted to be dominant, with basal slip also making a significant contribution.

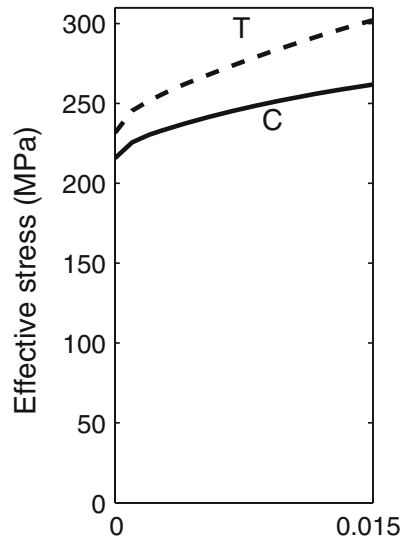

(a)

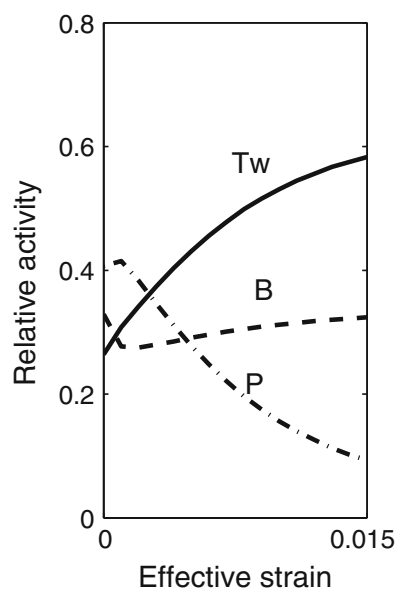

(b)

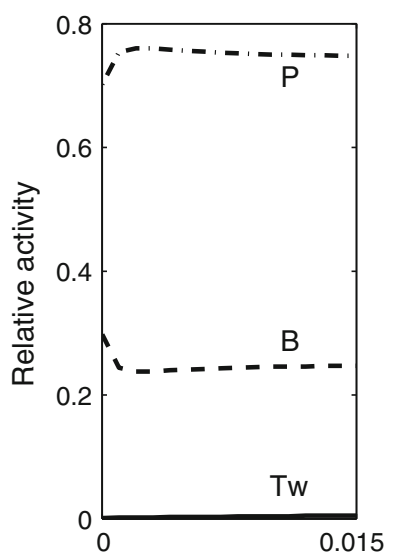

(c)

Fig. 8-(a) Predicted stress-strain response for deformation in compression (C) and tension (T) for an ideal precipitate distribution for asymmetry reduction (basal plates, volume fraction 10 pct, and equivalent precipitate size 50 nm). Predicted relative deformation system activity in $(b)$ compression and $(c)$ tension. 


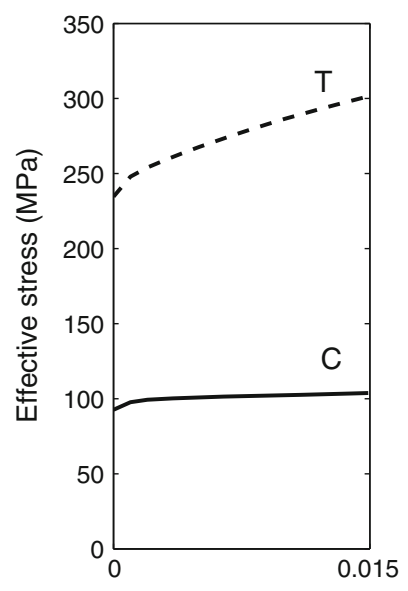

(a)

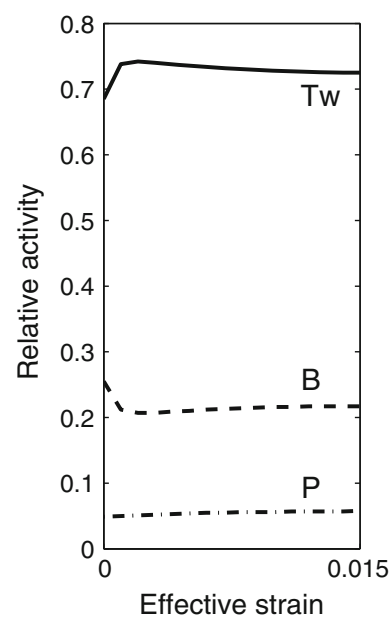

(b)

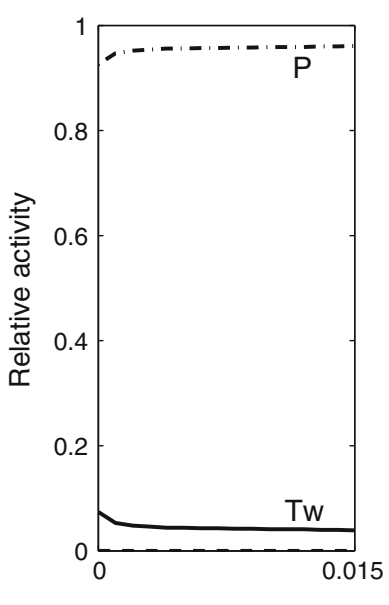

(c)

Fig. 9-(a) Predicted stress-strain response for deformation in compression (C) and tension (T) for 3 pct volume fraction of c-axis rod precipitates, details in the text. Predicted relative deformation system activity in $(b)$ compression and $(c)$ tension.

It is noteworthy that the predicted flow stresses and the asymmetry ratio for this idealized case are similar to those measured in the AZ91 peak-aged extrusion (Figure 8(a)). The microstructure of aged AZ91 is complex and contains regions of both continuous and discontinuous precipitation, ${ }^{[46]}$ and so it is difficult to apply this analysis in a simple way to that alloy. However, the results of this prediction suggest that for the purpose of providing the maximum reduction in yield asymmetry, standard commercial AZ91 is close to the best performance that could be expected from exploitation of precipitation.

This ideal case can be compared with the simulated behavior in the case of c-axis rod-shaped precipitates as formed in Z5. For this simulation, the average particle parameters (length, diameter, and volume fraction) for the peak-aged condition and CRSS values before precipitation are taken from Reference 40 . The strengthening contributions for the different modes due to precipitates were calculated and added to the preprecipitation CRSS values. Note, no attempt has been made to fit the model or account for the effect of solute change on strengthening. The CRSS values derived from this analysis for basal slip, prismatic slip, and $\{10 \overline{1} 2\}$ twinning were 78,142 , and $26 \mathrm{MPa}$, respectively. As the purpose of this simulation is to isolate the effect of precipitates only, the same input texture as for all other simulations was used (the simulated basal texture as shown in Figure 1(a)) rather than the true Z5 texture reported in Reference 40.

Results from the simulation are shown in Figure 9. It can be seen that, as expected, the predicted asymmetry is much greater than that in the ideal case shown in Figure 8. Furthermore, the predictions show that asymmetry has become more marked than in a precipitate-free case (e.g., compare with Figure 1(a)). This simulation is consistent with the experimental results shown in Figure 7. Predicted deformation system activities in compression and tension are shown in Figure 9(b) and (c), respectively. The most striking difference between the predictions for the ideal case of basal plates (Figure 8) and c-axis rods is that c-axis rods do not reduce the activity of twinning in axial compression as these particles are predicted to be poor strengtheners against twin growth. Prismatic slip is predicted to dominate deformation in axial tension, which is the same as in the ideal case (Figure 8(c)).

Note that the Orowan calculations are very sensitive to the exact values used for particle size, aspect ratio, and assumptions about the distribution (e.g., random, triangular array, etc.) and thus care should be taken not to over-interpret the results of these simulations. Nevertheless, the general trends are correct and provide an understanding of the effect of precipitate's shape and habit on asymmetry, which is consistent with observations.

Finally, a common objective is to achieve both low asymmetry/anisotropy and maximum strength. As already demonstrated, although the basal plate precipitates are the most effective in inhibiting twin growth, they are poor strengtheners against basal slip, and so the overall alloy strength will be low in orientations that are favorable to activate basal slip. To meet the combined objective of minimum asymmetry and maximum strength, a mix of different precipitate types is therefore expected to be optimal. In particular, a mix of basal and prismatic plates can provide effective strengthening against both basal slip and twinning. The effect of having a mixed precipitate population is discussed in more detail elsewhere. ${ }^{9]}$

\section{CONCLUSIONS}

The effect of precipitate's shape and habit on the strengthening of the major slip and twinning modes in magnesium has been discussed. It has been predicted that by selecting an alloy composition to favor precipitates of a particular shape and habit, it is possible to tailor the strengthening to inhibit some deformation 
modes more strongly than others. This differential strengthening has been predicted to be a potential route to reduce asymmetry in strongly textured wrought magnesium products. The following conclusions can be drawn from this study:

1. To reduce yield asymmetry in strongly basal textured magnesium alloy extrusions, it is necessary to suppress $\{10 \overline{1} 2\}$ twinning without strongly inhibiting slip prismatic slip, so that slip can activate before twinning when loading both axial tension and compression.

2. It has been demonstrated that in a single crystal, yield asymmetry would only be eliminated by increasing the critical resolved shear stress for twinning by over 20 times without hardening against prismatic slip, and there is no apparent practical way to achieve this. However, crystal plasticity modeling of polycrystalline magnesium has predicted that, in polycrystals, the difference in strengthening required to activate slip before twinning is less and that basal slip can make a significant contribution to strain accommodation because of the tilt from the perfect basal texture exhibited by many grains in a polycrystalline assembly.

3. It has been proposed that precipitation provides the most promising route to obtain the differential strengthening needed to suppress $\{10 \overline{1} 2\}$ twinning as it has been demonstrated that precipitate's shape and habit can have a markedly different strengthening effect on different deformation modes in magnesium.

4. Orowan calculations have been performed to predict the average strengthening effect against basal and prismatic slip produced by basal plates, prismatic plates, and c-axis rods. Prismatic plates provide the greatest strengthening against basal slip, and basal plates the least. $\mathrm{C}$-axis rods and prismatic plates are potent strengtheners against prismatic slip, with basal plates again having the lowest strengthening effect.

5. Reorientation of the lattice in $\{10 \overline{1} 2\}$ twins leads to a markedly different precipitate strengthening effect in the twin compared with parent. In particular, basal plates in the parent have a prismatic-like orientation in the twin and are predicted to be the most effective strengtheners against basal slip in the twin.

6. The additional stress required to activate basal slip in twinned material precipitation will directly influence the level of un-relaxed back-stress that inhibits twin growth. The strengthening against basal slip in the twin is therefore expected to directly correlate with the stress required for twin growth. Based on this analysis, basal plates are predicted to be the most effective in inhibiting twin growth and c-axis rods the least effective.

7. Based on the above arguments, it has been predicted that basal plates will lead to reduced asymmetry as they lead to the strongest inhibition of $\{10 \overline{1} 2\}$ twin growth but do not strengthen strongly against prismatic slip. C-axis rods have been predicted to potentially increase asymmetry by strengthening against prismatic slip much more strongly than twin growth.

8. The predictions of the model have been tested against mechanical data for alloys Z5 and AZ91 aged to peak strength and deformed in tension and compression. The precipitation of c-axis rods in Z5 produced an increase in asymmetry, whereas the precipitation of basal plates in AZ91 reduced asymmetry. This is in agreement with the model predictions.

9. Crystal plasticity modeling using realistic particle parameters suggests that an optimal distribution of basal plate precipitates will lead to near-complete elimination of yield asymmetry in astrongly textured magnesium extrusion.

\section{ACKNOWLEDGMENTS}

JDR thanks the UK Royal Academy of Engineering for funding this collaboration between the University of Manchester and Deakin University through the Global Research Award Scheme. J. Geng is thanked for facilitating transmission electron microscopy analysis.

\section{REFERENCES}

1. I.J Polmear: Light Alloys, 3rd ed., Arnold, London, 1995

2. M.R. Barnett, N. Stanford, P. Cizek, A. Beer, Z. Xuebin, and Z. Keshavarz: JOM, 2009, vol. 61, pp. 19-24.

3. T. Al-Samman and X. Li: Mater. Sci. Eng. A, 2011, vol. 528, pp. 3809-22.

4. K. Hantzsche, J. Bohlen, J. Wendt, K.U. Kainer, S.B. Yi, and D. Letzig: Scripta Mater., 2010, vol. 63, pp. 725-30.

5. J. Bohlen, M.R. Nürnberg, J.W. Senn, D. Letzig, and S.R. Agnew: Acta Mater., 2007, vol. 55, pp. 2101-22.

6. S. Suwas, G. Gottstein, and R. Kumar: Mater. Sci. Eng. A, 2007, vol. 471, pp. 1-14

7. J.F. Nie: Scripta Mater., 2003, vol. 48, pp. 1009-1015.

8. J.D. Robson, N. Stanford, and M.R. Barnett: Acta Mater., 2011, vol. 59, pp. 1945-56.

9. J.D. Robson: Proceedings of 9th International Conference on Magnesium Alloys and Their Applications, 2012, pp. 63-67.

10. C.N. Tomé, R.A. Lebensohn, and U.F. Kocks: Acta Metall. Mater., 1991, vol. 39, pp. 2667-80.

11. C.N. Tomé: Model. Simul. Mater. Sci. Eng., 1999, vol. 7, pp. 723-738.

12. C.N. Tomé, P.G. Maudlin, R.A. Lebensohn, and G.C. Kaschner: Acta Mater., 2001, vol. 49, pp. 3085-96.

13. S.R. Agnew, C.N. Tomé, D.W. Brown, T.M. Holden, and S.C. Vogel: Scripta Mater., 2003, vol. 48, pp. 1003-08.

14. S. Agnew, D. Brown, and C.N. Tomé: Acta Mater., 2006, vol. 54, pp. 4841-52.

15. O. Muránsky, D.G. Carr, M.R. Barnett, E.C. Oliver P. Sittner: Mater. Sci. Eng. A, 2008, vol. 496, pp. 14-24.

16. W.B. Hutchinson and M.R. Barnett: Scripta Mater., 2010, vol. 63, pp. $737-40$.

17. B.C. Wonseiwicz and W.A. Backofen: Trans. Am. Inst. Min. Eng., 1967, vol. 239, p. 1422.

18. H. Wang, B. Raeisinia, P.D. Wu, S.R. Agnew, and C.N. Tomé: Int. J. Solids Struct., 2010, vol. 47, pp. 2905-17.

19. M.R. Barnett: Proceedings of 9th International Conference on Magnesium Alloys and their Applications, 2012, pp. 1-4.

20. J. Wang, J.P. Hirth, and C.N. Tomé: Acta Mater., 2009, vol. 57, pp. $5521-30$ 
21. J. Wang, I. Beyerlein, and C.N. Tomé: Scripta Mater., 2010, vol. 63 , pp. $741-46$.

22. I.J. Beyerlein and C.N. Tomé: Proc. R. Soc. A, 2010, vol. 466, pp. 2517-44.

23. C.N. Tomé, I.J. Beyerlein, J. Wang, and R.J. McCabe: JOM, 2011, vol. 63, pp. 19-23.

24. J.W. Christian and S. Mahajan: Prog. Mater. Sci., 1995, vol. 39, pp. 1-157.

25. H. El Kadiri and A.L. Oppedal: J. Mech. Phys. Solids, 2010, vol. 58, pp. 613-24.

26. A.L. Oppedal, H. El Kadiri, C.N. Tomé, G.C. Kaschner, S.C. Vogel, J.C. Baird, and M.F. Horstemeyer: Int. J. Plasticity, 2012, vols. 30-31, pp. 41-61.

27. M.R. Barnett, A. Sullivan, N. Stanford, N. Ross, and A. Beer: Scripta Mater., 2010, vol. 63, pp. 721-24.

28. S.R. Agnew, M.H. Yoo, and C.N. Tomé: Acta Mater., 2001, vol. 49 , pp. $4277-89$

29. S.S. Park, B.S. You, and D.J. Yoon: J. Mater. Process. Technol., 2009, vol. 209, pp. 5940-43.

30. A. Akhtar and E. Teghtsoonian: Acta Metall. Mater., 1969, vol. 17 , pp. $1351-56$.

31. A.H. Blake and C.H. Caceres: Mater. Sci. Eng. A, 2008, vols. $483-$ 484, pp. 161-63.

32. J.F. Nie, B.C. Muddle, and I.J. Polmear: Mater. Sci. Forum, 1996, vols. 217-222, pp. 1257-62.
33. J.F. Nie and B.C. Muddle: J. Phase Equilib., 1998, vol. 19, pp. 543-51.

34. A.W. Zhu and E.A. Starke: Acta Mater., 1999, vol. 47, pp. $3263-3269$.

35. J.D. Embury and D.J. Lloyd: Treatise on Materials Science and Technology, vol. 31, A.K. Vasudevan and R.D. Doherty, eds., Academic Press, London, 1989, pp. 579-601.

36. R.L. Fullman: TMS-AIME, 1953, vol. 197, pp. 447-50.

37. J.B. Clark: Acta Metall. Mater., 1965, vol. 13, pp. 1281-89.

38. J.B. Clark: Acta Metall. Mater., 1968, vol. 16, pp. 141-151.

39. M.A. Gharghouri, G.C. Weatherly, and J.D. Embury: Philos. Mag. A, 1998, vol. 78, pp. 1137-49.

40. N. Stanford and M.R. Barnett: Mater. Sci. Eng. A, 2009, vol. 516, pp. 226-34.

41. J. Jain, W.J. Poole, C.W. Sinclair, and M.A. Gharghouri: Scripta Mater., 2010, vol. 62, pp. 301-04.

42. J. Geng, Y.B. Chun, N. Stanford, C.H.J. Davies, J.F. Nie, and M.R. Barnett: Mater. Sci. Eng. A, 2011, vol. 528, pp. 3659-65.

43. N. Stanford, J. Geng, Y.B. Chun, C.H.J. Davies, J.F. Nie, and M.R. Barnett: Acta Mater., 2012, vol. 60, pp. 218-28.

44. J.D. Robson, N. Stanford, and M.R. Barnett: Scripta Mater., 2010, vol. 63, pp. 823-26.

45. D. Wenwen, S. Yangshan, M. Xuegang, X. Feng, Z. Min W. Dengyun: Mater. Sci. Eng. A, 2003, vol. 356, pp. 1-7.

46. M.X. Zhang and P.M. Kelly: Scripta Mater., 2003, vol. 48, pp. $647-52$. 